\title{
Preoperative prediction of hepatocellular carcinoma with highly aggressive characteristics using quantitative parameters derived from hepatobiliary phase MR images
}

\author{
Zheng Ye ${ }^{1 \#}$, Likun Cao ${ }^{1,2 \#}$, Yi Wei ${ }^{1}$, Jie Chen ${ }^{1}$, Zhen Zhang ${ }^{1}$, Shan Yao ${ }^{1}$, Ting Duan ${ }^{3}$, Bin Song ${ }^{3}$ \\ ${ }^{1}$ West China School of Medicine, Sichuan University, Chengdu 610041, China; ${ }^{2}$ Department of Radiology, Peking Union Medical College Hospital \\ (Dongdan Campus), Beijing 100730, China; ${ }^{3}$ Department of Radiology, West China Hospital, Sichuan University, Chengdu 610041, China \\ Contributions: (I) Conception and design: Z Ye, B Song; (II) Administrative support: B Song; (III) Provision of study material or patients: L Cao; \\ (IV) Collection and assembly of data: Z Ye, L Cao; (V) Data analysis and interpretation: Z Ye, Y Wei, J Chen; (VI) Manuscript writing: All authors; \\ (VII) Final approval of manuscript: All authors. \\ \#These authors contributed equally to this work. \\ Correspondence to: Bin Song. Department of Radiology, West China Hospital, Sichuan University, No. 37 Guoxue Alley, Wuhou District, Chengdu \\ 610041, China. Email: songlab_radiology@163.com.
}

Background: To prospectively determine whether the quantitative imaging parameters derived from the hepatobiliary phase (HBP) can be used for the preoperative prediction of hepatocellular carcinoma (HCC) with highly aggressive characteristics.

Methods: One hundred and three patients with surgical-proven HCC were included from July 2015 to June 2018. Two independent reviewers measured signal intensity (SI) of liver and tumor, and quantitative parameters, including relative tumor enhancement (RTE), tumor to liver contrast ratio (TLR), tumor enhancement index (TEI), and relative enhancement ratio (RER) were calculated. The aggressive characteristics of HCC were identified by using the Ki-67 labeling index (LI), and patients were classified into low aggressive (Ki-67 LI $\leq 10 \%$ ) and high aggressive (Ki-67 LI >10\%) groups. The difference of quantitative parameters between two groups was assessed, and the correlation between quantitative parameters and Ki-67 LI was explored. Receiver operating characteristic analyses was used to evaluate the predictive performance of quantitative parameters.

Results: The values of RTE, TLR, TEI, and RER, were significantly lower in the highly aggressive group than low aggressive group $(\mathrm{P}<0.05)$, and negative correlations were obtained between these quantitative parameters and Ki-67 LI ( $r$ ranges from -0.41 to $-0.22, \mathrm{P}<0.05$ ). TLR demonstrated the highest predictive performance with the area under curve (AUC) of 0.83 [95\% confidence interval (CI): 0.75-0.90], sensitivity of $89.0 \%$ and specificity of $63.3 \%$, and subsequent with RER, TEI, and RTE with AUC of 0.78 (95\% CI: 0.68-0.85), 0.74 (95\% CI: 0.64-0.82) and 0.68 (95\% CI: 0.58-0.77), respectively. Good inter-observer and intra-observer agreement were found in all parameters.

Conclusions: TLR showed the highest predictive performance in highly aggressive HCC. Quantitative parameters based on HBP could preoperatively predict the aggressiveness of HCC.

Keywords: Hepatocellular carcinoma (HCC); Ki-67 antigen; quantitative analysis; hepatobiliary phase (HBP); sensitivity and specificity

Submitted Nov 12, 2019. Accepted for publication Dec 20, 2019.

doi: $10.21037 /$ atm.2020.01.04

View this article at: http://dx.doi.org/10.21037/atm.2020.01.04 


\section{Introduction}

Hepatocellular carcinoma (HCC) is the world's second leading cause of cancer-related death with the rapidly increasing incidence over the past two decades $(1,2)$. Liver transplantation, hepatectomy, transcatheter arterial chemoembolization (TACE) and radiofrequency ablation (RFA) are known to be potentially curative (3). Nevertheless, in HCCs with different biological characteristics, the treatment efficacy and prognosis differed significantly $(4,5)$. HCCs with highly aggressive properties typically proliferate rapidly and frequently with recurrence (6), resulting in poor prognosis.

Clinically speaking, patients with highly aggressive HCCs are often encouraged to receive preoperative adjuvant therapy, such as preoperative TACE, to improve their prognosis (1-3). The cell proliferation index, Ki-67 labeling index (LI), often assesses the aggressiveness of HCC, which was a stable immunohistochemical marker and defined as the percentage of Ki-67 positively stained cells of a given cell population, as $\mathrm{Ki}-67$ protein only expresses in the cell cycle of $G_{1}, S, G_{2}$, and mitosis except for $\mathrm{G}_{0}$, indicating active cell proliferation and invasive potential (4). Ki-67 LI has been reported as an independent prognostic indicator of HCC in previous studies (5-7). Cao et al. revealed that aggressive HCCs with high level of Ki-67 LI showed significant lower recurrence-free survival (RFS) rate and overall survival rate after surgery than those with low Ki-67 LI (5). Our previous study also reported the similar results that HCCs with high aggressiveness and high Ki-67 LI recurred easily within 1 year after surgery (8). However, pathological methods are currently used as reference standards to assess Ki-67 $\mathrm{LI}$, which is invasive and prone to sampling variability. Therefore, preoperative and noninvasive assessment of tumor aggressiveness in HCC using Ki-67 LI is of immense importance to guide personalized treatment strategies in clinical practice. Recently, investigators have found that the whole-tumor magnetic resonance imaging (MRI) histogram-derived parameters and texture analysis can be used for the prediction of Ki-67 LI in HCC patients $(9,10)$. However, the direct correlation between histogramderived parameters and the pathophysiologic process remains inconclusive. In addition, qualitative imaging features (i.e., arterial inhomogeneous enhancement) can also be used for predicting Ki-67 LI (11), but such qualitative parameter cannot be quantitatively described and it entails inter- observer bias. Thus, a more reliable and quantitative method is needed to predict Ki-67 LI in patients with HCC.

MRI examination with a liver-specific contrast agent, gadolinium ethoxybenzyl dimeglumine (Gd-EOB-DTPA), enables a noninvasive and comprehensive evaluation of HCC lesions and functional assessment of hepatocytes with hepatobiliary phase (HBP) (12). During this examination, normal hepatocytes uptake the contrast agent via organic anion transporter polypeptides (OATP) and excrete it through the biliary system (13). In HCC without or with partially functioning hepatocytes, the expression of OATP1B1/B3 is usually decreased (or absent), while multidrug resistance-associated proteins 2 (MRP2) expression is often increased, thereby demonstrating as hypointense lesions when compared with background liver on HBP $(13,14)$. Although most of HCC presented hypointensity on HBP, the absolute signal intensity (SI) and relative contrast enhancement ratio are not the same (15). Several studies showed that quantitative calculations with tumoral SI on HBP could predict the Edmondson-Steiner (E-S) grades of HCC $(16,17)$, and the relative enhancement ratio was a significant predictive factor in E-S grade IV (18). Fujita $e t a l$. also found that HCC with higher tumor to liver SI ratio on HBP demonstrated significantly higher OATP expression and better prognosis (19). Therefore, the purpose of this study is to prospectively determine whether the quantitative imaging parameters derived from HBP can be used for the preoperative prediction of hepatocellular carcinoma (HCC) with highly aggressive characteristics.

\section{Methods}

\section{Patients}

The Institutional Review Board of West China Hospital approved this prospective study, and all patients provided written informed consent. Inclusion criteria were patients with (I) a minimum age of 18 years old; (II) focal liver lesions suspected of malignant tumors on the basis of medical history and previous computed tomography (CT)/ ultrasonography examinations; (III) no previous antitumoral treatment (i.e., no RFA, TACE or hepatectomy) before GdEOB-DTPA enhanced MRI examination; (VI) Child-Pugh class A or B and surgical resection was recommended at our hospital. Patients with non-HCC tumors or inadequate clinical, imaging, and pathological information were excluded (Figure 1). 


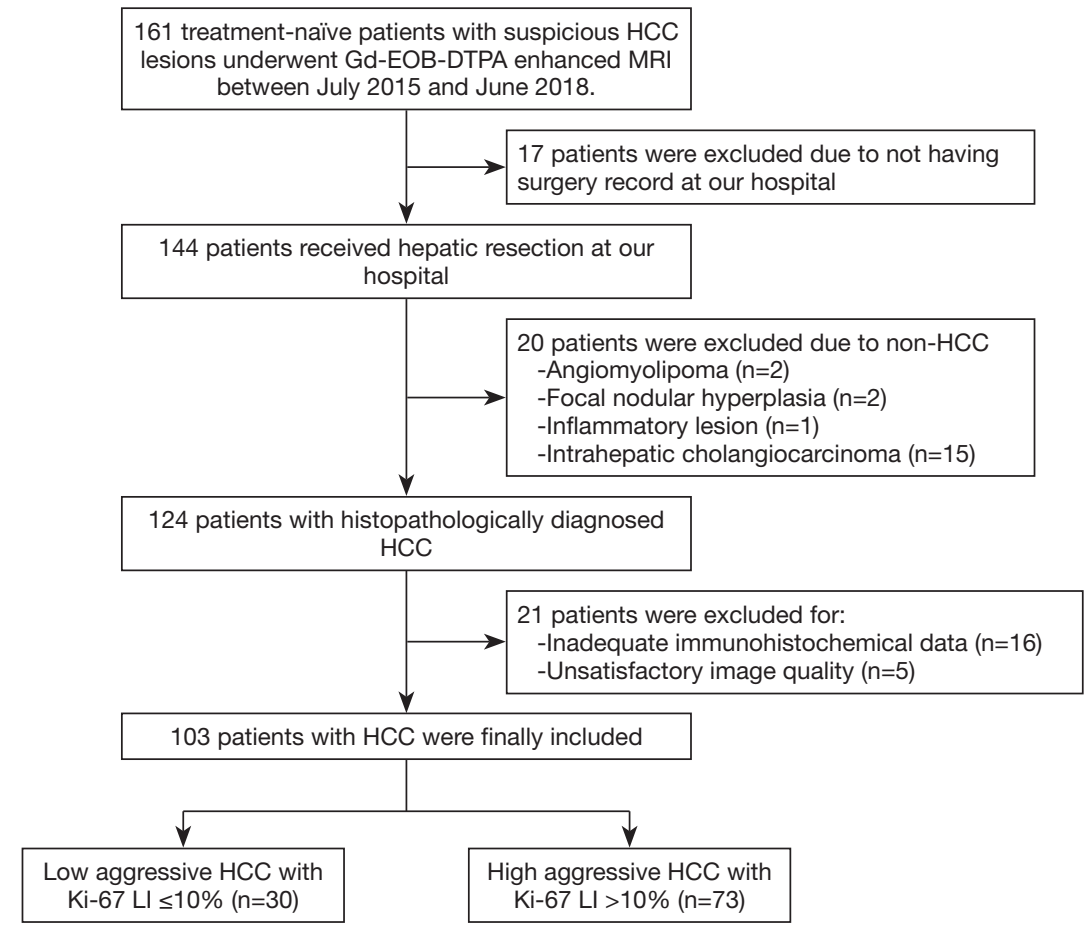

Figure 1 The flow chart illustrates the patient inclusion and exclusion process for the study cohort. HCC, hepatocellular carcinoma.

\section{MRI examination}

All MRI examinations were performed on a 3.0T MR scanner (MAGNETOM Skyra, Siemens Healthcare, Erlangen, Germany) with an 18-channel phased torso coil. All patients were required to fast at least 6 hours before the MRI examination. Pre-enhancement (PRE) scan, dynamic contrast-enhanced phases, and HBP were acquired with a T1-weighted volume interpolated breath hold (VIBE) gradient recall echo sequence. A dose of $0.025 \mathrm{mmol} / \mathrm{kg}$ Gd-EOB-DTPA (Primovist ${ }^{\circledR}$; Bayer Schering Pharma AG) was injected at a rate of $2.0 \mathrm{~mL} / \mathrm{s}$ followed by a $30-\mathrm{mL}$ saline flush at the same rate. The test bolus method was used to determine the timing of the arterial phase (AP), while the portal venous phase (PVP), transitional phase (TP), and HBP were acquired in 1, 3, and 20 minutes after Gd-EOB-DTPA administration, respectively. The T1weighted in/out phase, T2-weighted turbo spin echo (TSE), and diffusion-weighted imaging (DWI) sequences were also conducted. Table 1 shows the detailed parameters of each sequence.

\section{Quantitative analysis}

All quantitative measurements of SI were performed on the picture archiving and communications system (PACS) by two abdominal radiologists (Likun Cao and Yi Wei with 4 and 10 years of experience in abdominal MRI). The observers were blinded to the other imaging results, any clinical information, and the final pathological diagnoses, and the first radiologist measured the SI again after one month. For liver parenchyma, three circle regions of interest (ROIs) with $3 \mathrm{~cm}^{2}$ area were respectively placed on the left lobe, right lobe anterior and posterior segments, avoiding major vessels, tumor, and bile ducts. For HCC lesion, three freehand ROIs with size ranging from 1.06 to $88.24 \mathrm{~cm}^{2}$ were drawn along the tumor margin at or next the level of the largest diameter of the tumor, excluding vessels and necrotic areas. The average SI of liver parenchyma and the tumor was recorded as $\mathrm{L}_{0}$ and $\mathrm{T}_{0}$ based on PRE, and as $\mathrm{L}_{20}$ and $\mathrm{T}_{20}$ based on HBP, accordingly. The quantitative parameters were then calculated as the following formulas.

- Relative tumor enhancement (RTE): $\frac{\left(T_{20}-T_{0}\right)}{T_{0}}$

- Tumor to liver contrast ratio (TLR): $\frac{T_{20}}{L_{20}}$

- Tumor enhancement index (TEI): $\frac{\left(T_{20} \div L_{20}\right)}{\left(T_{0} \div L_{0}\right)}$ 
Table 1 Detailed parameters of MRI sequences

\begin{tabular}{lcccc}
\hline Sequence & T1-weighted VIBE & T1-weighted in/out phase & T2-weighted TSE & DWI \\
\hline Time of repetition $(\mathrm{msec})$ & 3.95 & 81 & 2,160 & 5,600 \\
Time of echo $(\mathrm{msec})$ & 1.92 & 1.4 & 700 & 68 \\
Flip angle & $9 \circ$ & $40 \times 32.5$ & $43.3 \times 43.3$ & $30^{\circ}$ \\
Field of view $\left(\mathrm{cm}^{2}\right)$ & $40 \times 29.6$ & $352 \times 286$ & $320 \times 288$ & $380 \times 289$ \\
Scan matrix & $352 \times 256$ & 6 & 6 & $100 \times 76$ \\
Slice thickness $(\mathrm{mm})$ & 2 & No & Yes & 6 \\
Fat suppression & Yes & $/$ & $/$ & $0,200,700,1,400$ and 2,100 \\
b values $\left(\mathrm{s} / \mathrm{mm}^{2}\right)$ & $/$ & Yes
\end{tabular}

VIBE, volume interpolated breath hold; TSE, turbo spin echo; DWI, diffusion-weighted imaging.

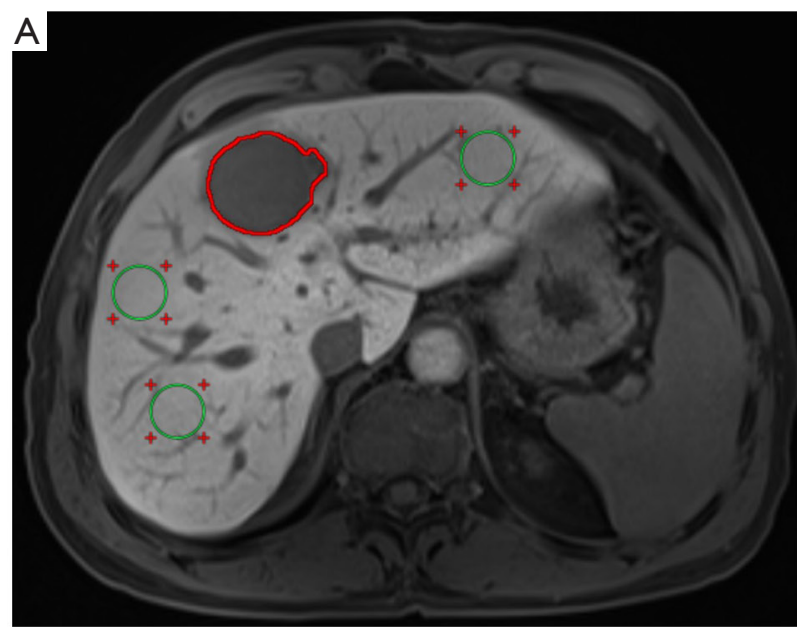

ROI placement

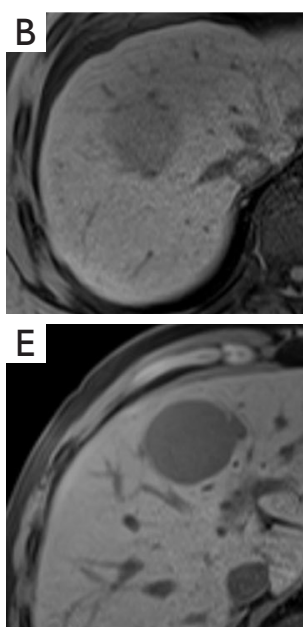

PRE

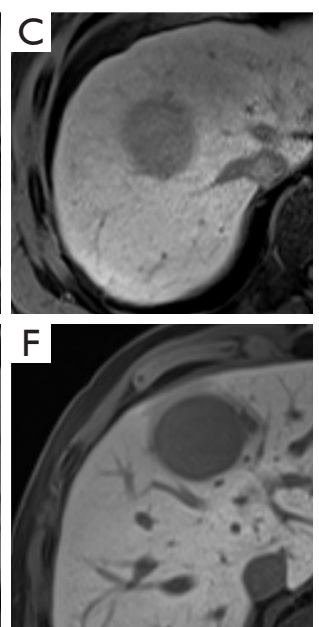

HBP

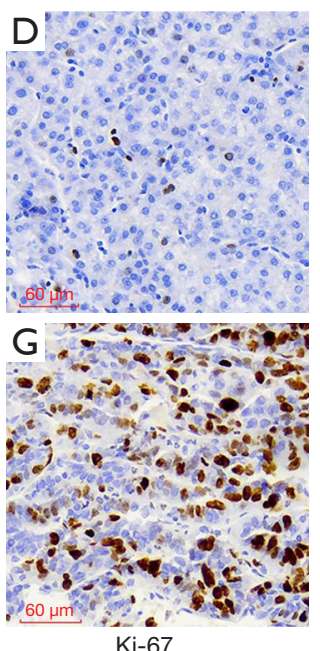

$\mathrm{Ki}-67$

Figure 2 Illustration of ROI placement (A) with three circles ROIs (green ROIs) on the left lobe, right lobe anterior and posterior segments, respectively, and a freehand ROI along the tumor margin at the level of the largest diameter of the tumor (red ROI). And the representative images of PRE, HBP, and Ki-67 staining (magnification, ×200) in low aggressive HCC (B,C,D) and high aggressive HCC (E,F,G). The RTE, TLR, TEI and RER were 0.32, 0.76, 0.93 and 0.62 in a 59-year-old male patient with low aggressive HCC (Ki-67 LI $=3 \%$ ) and $0.21,0.50,0.62$ and 0.18 in a 50-year-old male patient with high aggressive HCC (Ki-67 LI =57.62\%). ROI, region of interest; HCC, hepatocellular carcinoma; PRE, pre-enhancement; HBP, hepatobiliary phase; RTE, relative tumor enhancement; TLR, tumor to liver contrast ratio; TEI, tumor enhancement index; RER, relative enhancement ratio.

- Relative enhancement ratio (RER): $\frac{\left(T_{20}-T_{0}\right)}{\left(L_{20}-L_{0}\right)}$

The representative images with ROI placement and quantitative measurements were demonstrated in Figure 2.

\section{Immunobistochemical assessment}

Resected tumor sections were matched as closely as possible to the most significant section of the lesion, where the
SIs of the tumor were measured. All tumor tissues were fixed with $10 \%$ paraformaldehyde solution, and paraffinembedded, then cut into at $4 \mu \mathrm{m}$ for histopathological diagnosis of HCC, classification of E-S grade, the diagnosis of microvascular invasion (MVI) and immunohistochemical assessment. Ki-67 (antibody: Rabbit monoclonal, SP6, Abcam), cytokeratin 19 (CK19) (antibody: ab52625, Abcam) and epithelial cell adhesion molecule (EpCAM) (antibody: TA506627, Origene) staining was performed 
by standard avidin-biotin-peroxidase complex method and 3,3'-diaminobenzidine (DAB) solution was used for color development. The histopathological and immunohistochemical evaluation was performed by an experienced pathologist who was blinded to all imaging and clinical results. The classification of E-S grade, MVI presence, CK19, and EpCAM expression was the same as previous protocols or studies $(20,21)$. When Ki-67 LI was identified, HCC patients were classified into low aggressive (Ki-67 LI $\leq 10 \%$ ) and high aggressive (Ki-67 LI >10\%) groups according to previous research $(9,11)$.

\section{Statistical analysis}

The normality test firstly examined continuous variates and presented as means \pm standard deviations (SD) if normally distributed or median and interquartile ranges if not normally distributed. Categorical variates were presented as numbers and percentages. The difference of quantitative parameters between the low and high aggressive groups was assessed by the Student $t$-test or Mann-Whitney-Wilcoxon test, where appropriate. Spearman or Pearson correlation analysis with correlation coefficient rho $(r)$ was performed to evaluate the association between each quantitative parameter and Ki-67 LI. Diagnostic performance was assessed by receiver operating characteristic (ROC) analysis and the area under ROC curves (AUC). The comparison of different AUCs was conducted by the method described by DeLong et al. (22). Sensitivity and specificity were then determined at the optimal cutoff value selected by the Youden index. The intraclass correlation coefficient (ICC) and the Bland-Altman plots were illustrated to evaluate the inter-observer and intra-observer agreement of quantitative parameters, and ICC values of $0.90-1.00$ were considered as excellent agreement; $0.75-0.90$ as good agreement; $0.5-0.75$ as moderate agreement and $0-0.50$ as poor agreement.

All statistical calculations were performed with SPSS software (version 24.0, SPSS Inc., Chicago, IL, USA), MedCalc statistical software (version 15.8; https://www. medcalc.org) and GraphPad Prism (version 7.0; https:// www.graphpad.com). All statistical tests were two-sided, and a $\mathrm{P}$ value of $<0.05$ was considered significant.

\section{Results}

\section{Patient characteristics}

From July 2015 to June 2018, 161 consecutive patients were initially enrolled. Among them, 58 subjects were excluded for the following reasons: (I) not having surgery record at our hospital ( $\mathrm{n}=17)$; (II) non-HCC tumors $(\mathrm{n}=20)$; (III) inadequate surgical tissue for reliable immunohistochemical diagnosis (n=16); (IV) unsatisfactory image quality due to breathing artifact $(n=5)$. Finally, a total of 103 patients with HCC were included, and basic information and laboratory test results of included patients were obtained from our hospital information system (HIS). The main patient characteristics were summarized in Table 2. In the included 103 HCC patients ( 80 men and 23 women; mean \pm SD age: $50.90 \pm 11.93$ years), $73(70.9 \%)$ of lesions were identified as high aggressiveness, while 30 (29.1\%) of which were classified into the low aggressive group. There was no significant difference in age, sex ratio, tumor size, and laboratory results between low and high aggressive groups in HCC patients $(\mathrm{P}>0.05)$, while significant differences were observed in histopathological characteristics between two groups $(\mathrm{P}<0.05)$.

\section{Difference of quantitative parameters in two groups and its correlation with Ki-67 LI}

The median time interval between MRI examination and surgery was 2 days (range, $0-8$ days). All quantitative calculations were based on SI measured by the first radiologist. Figure 3 demonstrates the difference of quantitative parameters in two groups. The median and interquartile ranges of RTE, TLR, TEI and RER were 0.40 (0.24), 0.64 (0.19), $0.82(0.17)$ and $0.40(0.37)$ in low aggressive group, and $0.28(0.17), 0.52(0.12), 0.70$ (0.09) and $0.26(0.13)$ in high aggressive group, respectively. These quantitative parameters were significantly lower in patients with high aggressive HCCs than those of low aggressive HCCs $(\mathrm{P}<0.05)$. Significant negative correlations were observed among the Ki-67 LI and RTE $(r=-0.22, \mathrm{P}=0.023)$, TLR $(r=-0.41, \mathrm{P}<0.001)$, TEI $(r=-0.31, \mathrm{P}=0.001)$ and RER $(r=-0.35, \mathrm{P}<0.001)$ in patients with HCC.

\section{Predictive performance of quantitative parameters}

The AUC, optimal cutoff value, sensitivity and specificity of all quantitative parameters are presented in Table 3 and Figure 4. Among the quantitative parameters, TLR showed the highest diagnostic ability in predicting high aggressive HCC with the AUC value of 0.83 [95\% confidence interval (CI): $0.75-0.90$ ], sensitivity of $89.0 \%$ (95\% CI: 79.5-95.1\%) and specificity of $63.3 \%$ (95\% CI: 43.9-80.1\%). TEI and 
Table 2 Baseline and histopathological characteristics in low and high aggressive groups

\begin{tabular}{|c|c|c|c|c|}
\hline Variable & Overall $(n=103)$ & Low aggressive group $(n=30)$ & High aggressive group $(n=73)$ & $P$ value \\
\hline Age (years) & $50.90 \pm 11.93$ & $52.03 \pm 10.83$ & $50.44 \pm 12.39$ & 0.540 \\
\hline Sex (men/women) & $80 / 23$ & $25 / 5$ & $55 / 18$ & 0.376 \\
\hline Tumor size $(\mathrm{cm})$ & $5.79 \pm 2.82$ & $5.48 \pm 2.37$ & $5.92 \pm 3.00$ & 0.476 \\
\hline AST $(I U / L)^{a}$ & 37 [25] & 32 [25] & 39 [27] & 0.336 \\
\hline TBIL (umol/L) $)^{a}$ & $13.7[7.9]$ & $13.6[10.3]$ & $13.8[7.0]$ & 0.859 \\
\hline ALB (g/L) & $42.88 \pm 3.88$ & $43.78 \pm 3.79$ & $42.5 \pm 3.89$ & 0.129 \\
\hline AFP (ng/mL) & & & & 0.169 \\
\hline \multicolumn{5}{|c|}{ Histopathological characteristics } \\
\hline E-S grade & & & & 0.001 \\
\hline I & $19(18.4 \%)$ & $15(50.0 \%)$ & $4(5.5 \%)$ & \\
\hline II & $43(41.7 \%)$ & $8(26.7 \%)$ & $35(47.9 \%)$ & \\
\hline III & $21(20.4 \%)$ & $5(16.7 \%)$ & $16(21.9 \%)$ & \\
\hline IV & $20(19.4 \%)$ & $2(6.7 \%)$ & $18(24.7 \%)$ & \\
\hline MVI & & & & 0.032 \\
\hline Negative & $45(43.7 \%)$ & $18(60.0 \%)$ & 27 (37.0\%) & \\
\hline Negative & 73 (70.9\%) & 28 (93.3\%) & 45 (61.6\%) & \\
\hline Positive & 30 (29.1\%) & $2(6.7 \%)$ & 28 (38.4\%) & \\
\hline
\end{tabular}

${ }^{\text {a }}$, data are median values and data in square parentheses are interquartile ranges. ALT, alanine aminotransferase; AST, aspartate aminotransferase; TBIL, total bilirubin; ALB, albumin; AFP, alpha-fetoprotein; E-S grade, Edmondson-Steiner grade; MVI, microvascular invasion; CK19, cytokeratin 19; EpCAM, epithelial cell adhesion molecule.

RER also demonstrated good predictive performance with AUCs of 0.74 (95\% CI: 0.64-0.82) and 0.78 (95\% CI: $0.68-0.85$ ), sensitivity of $78.1 \%$ (95\% CI: $66.9-86.9 \%$ ) and $79.5 \%$, respectively, and same specificity of $63.3 \%$ (95\% CI: 43.9-80.1\%). Moderate predictive performance was also observed in RTE with AUC of 0.68 (95\% CI: 0.58-0.77), sensitivity of $64.4 \%$ (95\% CI: $52.3-75.3 \%$ ) and specificity of $70.0 \%$ (95\% CI: $50.6-85.3 \%)$.

\section{Inter-observer and intra-observer agreement}

The ICC values of RTE, TLR, TEI and RER were 0.89 (95\% CI: 0.84-0.92), 0.88 (95\% CI: 0.83-0.92), 0.85 (95\% CI: $0.79-0.90)$ and 0.87 (95\% CI: 0.81-0.91), respectively, between two radiologists; and 0.92 (95\% CI: 0.89-0.95), 0.92 (95\% CI: $0.89-0.95), 0.83$ (95\% CI: $0.76-0.88)$ and 0.89 (95\% CI: 0.84-0.92), respectively, between the first and 

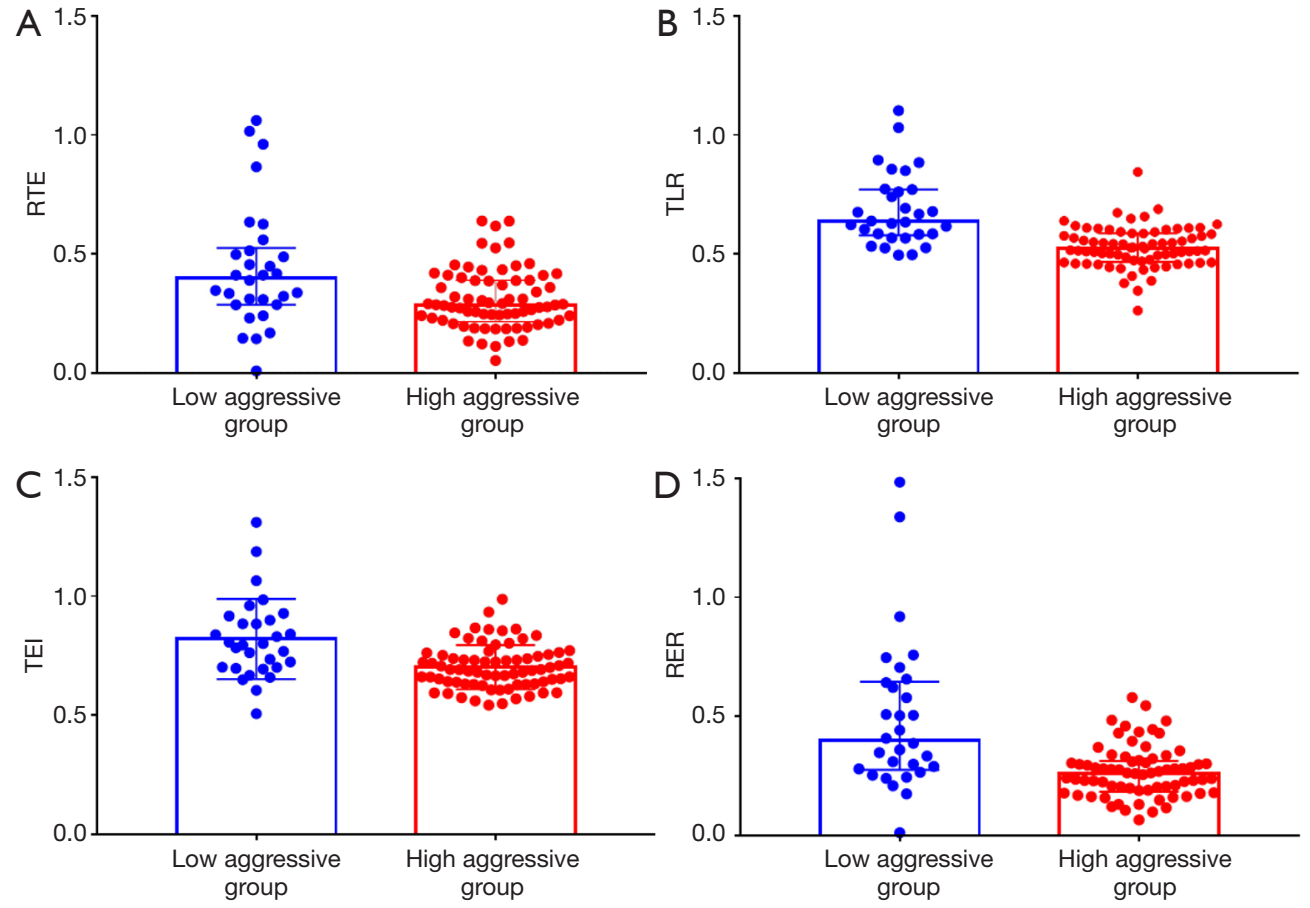

Figure 3 Box and whisker plots are the differences in RTE, TLR, TEI, and RER between low and high aggressive groups (A,B,C,D). These quantitative parameters are significantly lower in the highly aggressive group $(\mathrm{P}<0.05)$. RTE, relative tumor enhancement; TLR, tumor to liver contrast ratio; TEI, tumor enhancement index; RER, relative enhancement ratio.

Table 3 Predictive performance of quantitative parameters

\begin{tabular}{lcccc}
\hline Parameter & AUC $(95 \% \mathrm{Cl})$ & Optimal cutoff value & Sensitivity $(95 \% \mathrm{Cl})$ & Specificity $(95 \% \mathrm{Cl})$ \\
\hline RTE & $0.68(0.58-0.77)$ & $\leq 0.31$ & $64.4 \%(52.3-75.3 \%)$ & $70.0 \%(50.6-85.3 \%)$ \\
TLR & $0.83(0.75-0.90)$ & $\leq 0.61$ & $89.0 \%(79.5-95.1 \%)$ & $63.3 \%(43.9-80.1 \%)$ \\
TEl & $0.74(0.64-0.82)$ & $\leq 0.76$ & $78.1 \%(66.9-86.9 \%)$ & $63.3 \%(43.9-80.1 \%)$ \\
RER & $0.78(0.68-0.85)$ & $\leq 0.33$ & $79.5 \%(68.4-88.0 \%)$ & $63.3 \%(43.9-80.1 \%)$ \\
\hline
\end{tabular}

AUC, area under the ROC curve; RTE, relative tumor enhancement; TLR, tumor to liver contrast ratio; TEl, tumor enhancement index;

$\mathrm{RER}$, relative enhancement ratio; $\mathrm{Cl}$, confidence interval.

second measurement in the same radiologist (Likun Cao). The results together with Bland-Altman plots (Figure 5) suggested that there were good inter-observer and intraobserver agreement for all the parameters.

\section{Discussion}

In the present study, we found that RTE, TLR, TEI, and RER derived from HBP were significantly lower in high aggressive HCCs than those of low aggressive HCCs and showed a significant negative correlation with Ki-67 LI. In addition, moderate to excellent performance in predicting HCC with highly aggressive behavior was found in these quantitative parameters with AUCs range from 0.68 to 0.83 .

Highly aggressive HCC often requires adjuvant therapies before surgery to prolong the overall survival rates and RFS rates of patients (1-3). As a well-established proliferation marker, Ki-67 LI was reported to be highly correlated with the aggressive propensity of HCC and thus could affect the treatment response of the tumor and prognosis directly (4). Previous studies have shown that different histopathological and molecular manifestations of HCC often associated with 
each other (23), which agreed with our results that higher E-S grade, the presence of MVI, and progenitor phenotype were more frequently observed in highly aggressive HCCs with high Ki-67 LI. In addition, the staining and

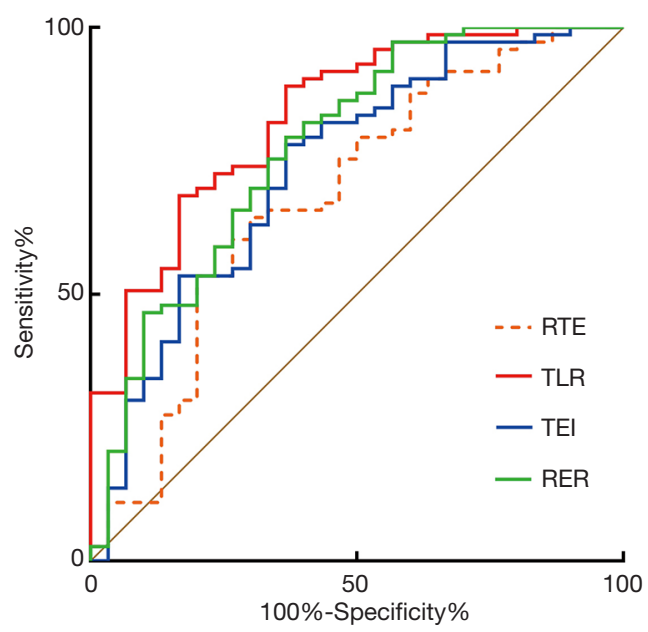

Figure 4 ROC curve of RTE, TLR, TEI, and RER derived from hepatobiliary phase for predicting HCC with highly aggressive behavior. The AUCs for predicting high aggressive HCC were 0.68 (95\% CI: 0.58-0.77) for RTE, 0.83 (95\% CI: 0.75-0.90) for TLR, 0.74 (95\% CI: $0.64-0.82)$ for TEI and 0.78 (95\% CI: 0.68-0.85) for RER. ROC, receiver operating characteristic; RTE, relative tumor enhancement; TLR, tumor to liver contrast ratio; TEI, tumor enhancement index; RER, relative enhancement ratio. interpretation of Ki-67 LI were considered to be relatively stable and reproducible among the immunohistochemical indexes of HCC (24). Hence, a preoperative and noninvasive method for prediction of tumor aggressiveness by using $\mathrm{Ki}-67 \mathrm{LI}$ is urgently needed.

Recently, Li et al. found that highly aggressive small HCC (sHCC) with Ki-67 LI higher than $10 \%$ demonstrated lower tumor-to-muscle relative enhancement ratio on AP, PVP and TP, which was following our findings that relative SI of HCC decreased with aggressiveness and Ki-67 LI increased (11). However, the significant difference between high and low Ki-67 LI group was not prominent in Li's study, probably due to the inevitable measurement errors in small lesions, as the average tumor size in their study was $2.04 \pm 0.65 \mathrm{~cm}$ for sHCC with low Ki-67 LI and $1.84 \pm 0.57 \mathrm{~cm}$ for sHCC with high Ki-67 LI. Besides, the contrast agent they used was gadobenic acid (Gd-BOPTA), and the possible role of HBP was not explored in their study. While in our study, the quantitative parameters RTE, TLR, TEI, and RER were based on the SI from both PRE and HBP, which may better reveal the biological behavior of HCC given the rationale of Gd-EOB-DTPA. What is more, others found that Ki-67 LI of HCC can be predicted by the histogram-derived parameters of $\mathrm{AP}$ and an apparent diffusion coefficient (ADC) map, or by the nomogram consisted of a radiomic signature and clinical features $(8,9)$. However, those methods often required whole-tumor delineation and sophisticated post-processing algorithms,
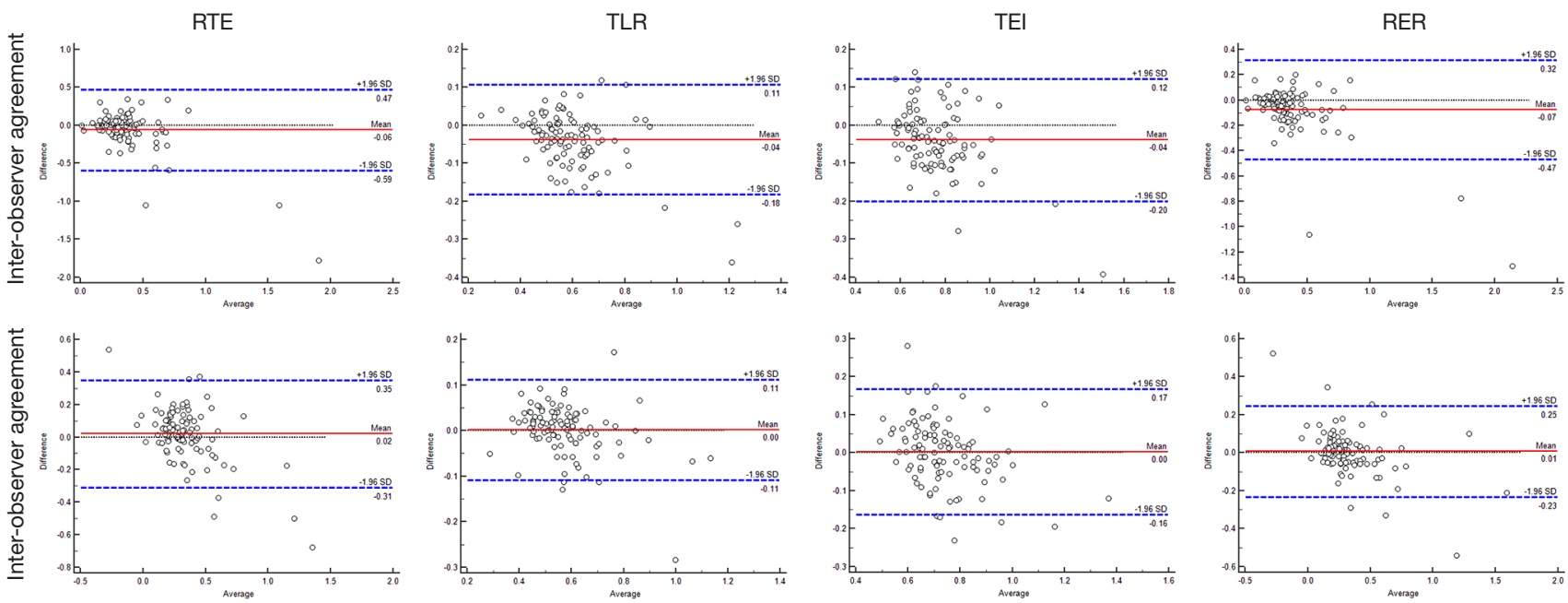

Figure 5 Bland-Altman plots of quantitative parameters for inter-observer and intra-observer agreement evaluation. The differences were relatively small. RTE, relative tumor enhancement; TLR, tumor to liver contrast ratio; TEI, tumor enhancement index; RER, relative enhancement ratio. 
and thus unavailable in clinical practice so far.

The SI of background liver on HBP is often influenced by liver function (25), however, all patients in our study were in Child-Pugh class A and had bilirubin levels within the normal ranges, therefore, the confounding effect of liver function to the SI of background hepatic parenchyma on HBP would be negligible (15). In the current study, we found that HCCs in the highly aggressive group showed significantly lower RTE, TLR, TEI, and RER, which agreed with previous studies that HCC with lower relative tumoral SI on HBP had more aggressive biological behavior, such as advanced tumor grades and shorter progression-free survival (PFS) rate. Jin et al. found that poorly differentiated HCC could be preoperatively identified by TEI, which was also reported to be one of the independent predictive indicators of HCC with E-S grade IV (18). Additionally, RER was found to be negatively correlated with HCC histological grades $(\mathrm{r}=-0.775, \mathrm{P}<0.001)$, probably due to increased expression of hepatocyte nuclear factor (HNF) $3 \beta$ in poorly-differentiated HCC down-regulating the expression of OATP1B3, leading to lower relative tumoral SI $(17,26)$. Moreover, HCC patients with lower preoperative TLR showed higher tumor grades and shorter PFS rates after hepatic arterial infusion chemotherapy (19). The reason why aggressive HCC tend to demonstrate lower relative tumoral SI probably is that normal hepatocytes gradually turns into uncontrolled and actively proliferated malignant tumor cells with higher Ki-67 LI during multistep hepatocarcinogenesis, while at the same time, the expression of OATP usually decreased, hence resulting in less uptake of Gd-EOB-DTPA. However, the association between the expression of $\mathrm{Ki}-67 \mathrm{LI}$ and OATP may need to be further investigated.

There are several limitations to our research. First, the sample size in our study, especially the low aggressive group was relatively small, as Gd-EOB-DPTA enhanced MRI was used only for research rather than routine clinical test at our hospital before 2019. However, as the number of patients increase in our hospital, future studies with a larger sample size will be conducted. Second, HCC with Ki-67 LI >10\% was determined as a highly aggressive tumor in our study. We defined this cutoff value based on previous studies, which reported that HCC patients with Ki-67 LI higher than $10 \%$ relapsed frequently, and the prognosis of these patients was poor $(5,9,11)$. However, there is currently no standardized threshold for Ki-67 LI in patients with HCC. Third, we measured the quantitative SI on the images from a 3.0 T MRI scanner. Therefore, our results may not apply to the $1.5 \mathrm{~T}$ MRI scanner. The quantitative measurement from scanners with different field strength and different MRI vendors are required to validate our results.

In conclusion, TLR showed the highest predictive ability in HCC with high aggressiveness. Thus, our results demonstrated that quantitative parameters based on the SI measurement of HBP could preoperatively and noninvasively predict the aggressive characteristics of HCC.

\section{Acknowledgments}

Funding: The study was supported by the Research Grant of National Nature Science Foundation of China (Grant number 81771797 ) and the 1.3 .5 project for disciplines of excellence, West China Hospital, Sichuan University (Grant number ZYJC18008).

\section{Footnote}

Conflicts of Interest: The authors have no conflicts of interest to declare.

Ethical Statement: The authors are accountable for all aspects of the work in ensuring that questions related to the accuracy or integrity of any part of the work are appropriately investigated and resolved. This prospective study was approved by the Institutional Review Board, and written informed consent was obtained from all patients This study followed the guidelines of diagnostic accuracy studies-STARD.

\section{References}

1. Bae SH, Park HC. Local modalities for inoperable hepatocellular carcinoma: radiofrequency ablation versus stereotactic body radiotherapy. Ann Transl Med 2018;6:S3.

2. Zhang YF, Guo RP, Zou RH, et al. Efficacy and safety of preoperative chemoembolization for resectable hepatocellular carcinoma with portal vein invasion: a prospective comparative study. Eur Radiol 2016;26:2078-88.

3. Wang $Y$, Shen $X$, Huang $S$, et al. Transcatheter arterial chemoembolization combined with elemene for the treatment of hepatic carcinoma. Transl Cancer Res 2018;7:1164-5.

4. Scholzen T, Gerdes J. The Ki-67 protein: from the known and the unknown. J Cell Physiol 2000;182:311-22. 
5. Cao Y, Ke R, Wang S, et al. DNA topoisomerase II $\alpha$ and $\mathrm{Ki} 67$ are prognostic factors in patients with hepatocellular carcinoma. Oncol Lett 2017;13:4109-16.

6. Guzman G, Alagiozian-Angelova V, Layden-Almer JE, et al. p53, Ki-67, and serum alpha feto-protein as predictors of hepatocellular carcinoma recurrence in liver transplant patients. Mod Pathol 2005;18:1498-503.

7. Li HH, Qi LN, Ma L, et al. Effect of KI-67 positive cellular index on prognosis after hepatectomy in Barcelona Clinic Liver Cancer stage A and B hepatocellular carcinoma with microvascular invasion. Onco Targets Ther 2018;11:4747-54.

8. Ye Z, Jiang H, Chen J, et al. Texture analysis on gadoxetic acid enhanced-MRI for predicting Ki-67 status in hepatocellular carcinoma: A prospective study. Chin J Cancer Res 2019;31:806-17.

9. Hu XX, Yang ZX, Liang HY, et al. Whole-tumor MRI histogram analyses of hepatocellular carcinoma: Correlations with Ki-67 labeling index. J Magn Reson Imaging 2017;46:383-92.

10. Li Y, Yan C, Weng S, et al. Texture analysis of multi-phase MRI images to detect expression of Ki67 in hepatocellular carcinoma. Clin Radiol 2019;74:813.e19-813.e27.

11. Li Y, Chen J, Weng S, et al. Small hepatocellular carcinoma: using MRI to predict histological grade and Ki-67 expression. Clin Radiol 2019;74:653.e1-653.e9.

12. Thian YL, Riddell AM, Koh DM. Liver-specific agents for contrast-enhanced MRI: role in oncological imaging. Cancer Imaging 2013;13:567-79.

13. Van Beers BE, Pastor CM, Hussain HK. Primovist, Eovist: what to expect? J Hepatol 2012;57:421-9.

14. Ringe KI, Husarik DB, Sirlin CB, et al. Gadoxetate disodium-enhanced MRI of the liver: part 1, protocol optimization and lesion appearance in the noncirrhotic liver. AJR Am J Roentgenol 2010;195:13-28.

15. Choi JY, Kim MJ, Park YN, et al. Gadoxetate disodiumenhanced hepatobiliary phase MRI of hepatocellular carcinoma: correlation with histological characteristics. AJR Am J Roentgenol 2011;197:399-405.

16. Kogita S, Imai Y, Okada M, et al. Gd-EOB-DTPAenhanced magnetic resonance images of hepatocellular carcinoma: correlation with histological grading and portal blood flow. Eur Radiol 2010;20:2405-13.

17. Tong HF, Liang HB, Mo ZK, et al. Quantitative analysis of gadoxetic acid-enhanced magnetic resonance imaging predicts histological grade of hepatocellular carcinoma.
Clin Imaging 2017;43:9-14.

18. Jin YJ, Cho SG, Lee KY, et al. Association between relative liver enhancement on gadoxetic acid enhanced magnetic resonance images and histologic grade of hepatocellular carcinoma. Medicine (Baltimore) 2017;96:e7580.

19. Fujita N, Nishie A, Asayama Y, et al. Significance of the Signal Intensity of Gadoxetic Acid-enhanced MR Imaging for Predicting the Efficacy of Hepatic Arterial Infusion Chemotherapy in Hepatocellular Carcinoma. Magn Reson Med Sci 2016;15:111-20.

20. Cao L, Chen J, Duan T, et al. Diffusion kurtosis imaging (DKI) of hepatocellular carcinoma: correlation with microvascular invasion and histologic grade. Quant Imaging Med Surg 2019;9:590-602.

21. Chen J, Wu Z, Xia C, et al. Noninvasive prediction of HCC with progenitor phenotype based on gadoxetic acidenhanced MRI. Eur Radiol 2020;30:1232-42.

22. DeLong ER, DeLong DM, Clarke-Pearson DL. Comparing the areas under two or more correlated receiver operating characteristic curves: a nonparametric approach. Biometrics 1988;44:837-45.

23. Calderaro J, Couchy G, Imbeaud S, et al. Histological Subtypes of Hepatocellular Carcinoma Are Related To Gene Mutations and Molecular Tumour Classification. J Hepatol 2017;67:727-38.

24. Yoshimoto J, Iwata T, Takamori S, et al. Usefulness of monoclonal antibody $\mathrm{Ki}-67$ as a prognostic factor of hepatocellular carcinoma. International Hepatology Communications 1997;6:209-18.

25. Cruite I, Schroeder M, Merkle EM, et al. Gadoxetate disodium-enhanced MRI of the liver: part 2, protocol optimization and lesion appearance in the cirrhotic liver. AJR Am J Roentgenol 2010;195:29-41.

26. Vavricka SR, Jung D, Fried M, et al. The human organic anion transporting polypeptide 8 (SLCO1B3) gene is transcriptionally repressed by hepatocyte nuclear factor 3 beta in hepatocellular carcinoma. J Hepatol 2004;40:212-8.

Cite this article as: Ye Z, Cao L, Wei Y, Chen J, Zhang Z, Yao S, Duan T, Song B. Preoperative prediction of hepatocellular carcinoma with highly aggressive characteristics using quantitative parameters derived from hepatobiliary phase MR images. Ann Transl Med 2020;8(4):85. doi: 10.21037/ atm.2020.01.04 